\title{
Relevance of epigenetic alterations of $H 19$ locus with androgen and estrogen in patients with C1-inhibitor deficiency
}

\author{
Sujoy Khan ${ }^{1}$ and Hilary Longhurst ${ }^{2}$ \\ ${ }^{1}$ Hull University Teaching Hospitals NHS Trust \\ ${ }^{2}$ Addenbrooke's Hospital
}

August 10, 2020

\begin{abstract}
The interesting report by Karagianni P et al on the finding of increased DNA methylation of H19 locus imprinting control region in saliva samples of Sjögren's syndrome patients correlating with low complement C4 levels, may offer insights into how $\mathrm{C} 4$ level may be regulated in serpinopathies such as C1-inhibitor deficiency. An undetectable or low C4 level in patients with severe angioedema is a feature of C1-inhibitor deficiency (hereditary angioedema (HAE) type I with low to absent function and antigenic levels; HAE type II with point mutations in SERPING1 gene that affect the reactive centre loop affecting protein function only). However, C4 levels do not always clinically correlate with disease activity, and up to $6 \%$ patients do not have known mutations in the SERPING1 gene.
\end{abstract}

\section{Hosted file}

CEI Commentary.doc available at https://authorea.com/users/349864/articles/474847-relevanceof-epigenetic-alterations-of-h19-locus-with-androgen-and-estrogen-in-patients-with-c1inhibitor-deficiency 